


\title{
Contribución de las unidades de información en las redes organizacionales
}

\author{
Peña Vera, Tania*
}

\section{Resumen}

Partiendo de una perspectiva racional, que luego llega a la abducción, se analiza la contribución de las unidades de información en las redes organizacionales generadoras de conocimiento; para esto se manejan algunos postulados teóricos que caracterizan y señalan tanto las funciones de las unidades de información en la actual sociedad globalizada, como el funcionamiento de las redes organizacionales. Como resultado se indica que las unidades de información contribuyen en los procesos de transferencia y creación de conocimiento que ocurre en dichas redes, en tanto que su acción impacta positivamente el desempeño de sus participantes. Se concluye primordialmente que las unidades de información propician el incremento del potencial creativo de los individuos que se manejan en ambientes redificados, por cuanto le proporcionan herramientas instrumentales tecnológico-analíticas para hacer un uso más provechoso de las fuentes de información, por lo que agregan valor a sus aportes intelectuales en la solución de problemas, el mejoramiento de procesos o la creación de innovaciones.

Palabras clave: Unidades de información, conocimiento, redes organizacionales, tecnología, alfabetización informacional.

Recibido: 05-07-01. Aceptado: 06-09-23

MSc. Docente de la Escuela de Bibliotecología y Archivología de la Universidad del Zulia (LUZ). Oficina ubicada en la Facultad de Humanidades y Educación.

E-mail: tanialirena@yahoo.com. 


\title{
The Contribution of Information Units in Organizational Networks
}

\begin{abstract}
Based on a rational perspective that becomes abduction, we can also analyze how information units help networks within organizational networks which produce knowledge. In order to do this certain extremely specific proposals or theories must be handled which characterize and indicate both the functions of information units in present day global society and the functioning of organizational networks. As a result, we say that these information units contribute to the transference processes and to the creation of knowledge that results from such networks in that their action positively affects the performance of its participants. To conclude, we can say basically that these information units promote an increase in the creative power of its participants who handle the network environment in that it offers some technological, analytical and instrumental tools in order to increase the benefits of the information sources and make them more valuable as intellectual benefits in problem solution, and in bettering the process of innovation creation.
\end{abstract}

Key works: Information units, knowledge, organizational networks, technology, information literacy.

\section{Introducción}

Dentro de los múltiples efectos derivados de la globalidad, está el incremento de la necesidad de compartir recursos y de enfatizar en el establecimiento de alianzas interorganizacionales, orientadas a integrar esfuerzos y obtener resultados más efectivos para los involucrados. En tal sentido, las redes organizacionales se constituyen como vías de acción que buscan primordialmente potenciar la capacidad de creativa de los individuos responsables de generar innovaciones.

En virtud del intercambio multidireccional y transdisciplinar producto del funcionamiento redificado, se facilita la transferencia y creación de conocimiento útil y necesario para agregar valor a las acciones desarrolladas en el interior de las organizaciones, las cuales ameritan elevar su capital intelectual así como conducir creativamente su participación en el mercado, a modo de remarcar y profundizar sus ventajas competitivas. Ante tal exigencia, el establecimiento de redes incorpora aportes de interés, más aún cuando son asistidas por unidades de información, es decir, entes especializados en la gestión de la información y el conocimiento, mediadores/facilitadores de este recurso esencial.

Las unidades de información promueven un uso más selectivo e inteligente de los contenidos, agregando valor a través de su validación, filtraje y facilitando la transformación de éstos en nuevos constructos cognitivos sintéticos, mediante el fomento de habilidades y destrezas en los usuarios de las redes. 
La presente investigación se plantea como objetivo general analizar la contribución de las unidades de información en las redes organizacionales generadoras de conocimiento. Inicia con la explicación del rol que desempeñan las unidades de información en la sociedad actual; seguido a esto, la conceptualización del funcionamiento de las redes organizacionales; y por último, se señala el aporte de las unidades de información en los procesos de transferencia y creación de conocimiento que se lleva a cabo en dichas redes.

Para el desarrollo de esta investigación se aplicó un enfoque híbrido en el sentido que en un primer momento se utilizó una vía racionalista -deductiva a través de la integración de las perspectivas teóricas que se analizaron, referidas al rol que desempeñan las unidades de información en el contexto actual y la conceptualización- caracterización de las redes organizacionales. Posteriormente, se aplicó la abducción, con el propósito de generar vínculos o coordenadas entre ambos temas, además de crear y fundamentar constructos conceptuales que vengan a llenar vacíos teóricos, y que sirvan a la vez como plataforma para expandir el conocimiento existente al respecto.

\section{Las unidades de información en el contexto actual}

Debido al uso intenso de las tecnologías de información y comunicación (en adelante TIC), las unidades de información se reconfiguran para poder "tener voz" en este nuevo contexto, para asistir de diversas formas las necesidades intelectivas de sus usuarios, quienes plantean interrogantes distintas y permeadas por la dinámica propia de estos tiempos en los que parece haber desaparecido la dimensión espacio-temporal, por cuanto ya no se está sujeto a horarios ni lugares para acceder a la información que se requiera.

Con el devenir de los cambios sociales, económicos y sobre todo tecnológicos sucedidos en las últimas décadas, las unidades de información han tenido que asumir transformaciones para estar a la par de la compleja realidad informativa, según Gazpio y Álvarez (1999), uno de esos aspectos tiene que ver con el crecimiento exponencial y la movilidad de la información experimentada en estos últimos años, porque si bien es cierto que han habido importantes avances en el ámbito tecnológico, lo cual permite procesar grandes volúmenes de información; la producción de contenidos tanto en formato digital como impreso, también se ha elevado exponencialmente y tiende a seguir aumentando (Kerckhove,1999; Villanueva, 1997). De esto se desprende que las unidades de información no puedan manejarse sin contar con una plataforma tecnológica, que incluya no sólo el equipamiento sino también el aprendizaje tecnológico necesario para operativizar adecuadamente dichas herramientas.

Por otra parte, en virtud de la acelerada obsolescencia de la información, resulta poco conveniente basarse en colecciones físicas que pierden relevancia y 
valor informativo en poco tiempo. Esto configura una nueva dimensión que es la desmaterialización de los soportes documentales, y por ende su creciente virtualización; ante ello, las unidades de información en la actualidad manejan cada vez más elementos inmateriales que pueden ser accedidos desde múltiples vías, su énfasis ya no es entonces la conservación, sino el hallazgo y análisis efectivo de contenidos.

Otro aspecto que de acuerdo con Gazpio y Álvarez (1999) incide en la caracterización de las unidades de información, es la globalización e instantaneidad que distingue a los medios de comunicación y los canales informativos, esto ha llevado a asumir un volumen inimaginable de información y conocimiento proveniente de cualquier latitud, y accedido además en cualquier momento. Este desdibujamiento de la dimensión espacio-temporal conduce a un replanteamiento en la tipología de los servicios de información que se prestan, porque ya no se circunscriben a un universo limitado de individuos, sino a una amplia pluralidad de usuarios para los que hay que diseñar servicios versátiles, mediante los cuales se puedan satisfacer sus necesidades cognitivas. Si en el esquema tradicional de las unidades de información, el usuario podía ser un individuo aislado o con pocos contactos, en este momento la comunicación se erige como elemento posibilitador en la prestación y recepción del servicio, repotenciando el intercambio no sólo del usuario con la unidad de información, sino también de éste con otros pares o entes en los que puede encontrar respuestas oportunas, y entre los diferentes servicios entre sí, dando lugar a la conformación de redes.

Esto lo confirma Almirón (2002) comparando la acumulación de capital (generación de riqueza) proveniente del acceso a la información y el derivado de los servicios de comunicación (conectividad), siendo éste último considerablemente mayor que el primero; además de la evolución experimentada por las tecnologías de información (manejo de contenidos) y las de comunicación (conectividad). Concluyendo que, aunque la información dota de poder, si no es posible usarla, conectarla con otros contenidos, comunicarla a terceros y explotarla a través de la comunicación, entonces esa información no posee valor.

Siguiendo con Gazpio y Álvarez (1999), otro de los rasgos sociales que caracteriza a las unidades de información es la especialización e interdisciplinariedad, esta deviene del hecho que la explosión de información a la que se asiste en las últimas décadas no sólo es cuantitativa sino también cualitativa, y que además existe una creciente profundización y diversificación en los diferentes campo del saber; por consiguiente, se hace énfasis en el desarrollo de habilidades de integración científica que conlleven por un lado, a conectar fuentes o constructos de conocimiento de diversos campos, y también a propiciar el encuentro de sujetos inmersos en áreas complementarias o semejantes. Ya que, las unidades de información han pasado de ser almacenes de información y conocimiento, a ser entes facilitadores y mediadores en 
el hallazgo y construcción de nuevos conocimientos.

De igual forma, la multiplicidad de medios es otro factor que permea a las unidades de información, por cuanto ha habido una diversificación de las fuentes de acceso al saber pero también de los medios para transmitirlo, esto conlleva a desarrollar la ergonomía en el servicio que se preste, es decir, formas cada vez más adaptadas a los estilos de representación mental de los individuos (visual, auditivo y kinestésico). De manera que las unidades de información confrontan cambios que atañen a la arquitectura de los documentos, sus contenidos, las características de sus usuarios, el tipo de servicio que suministran, incluyendo la orientación y los medios utilizados para ofrecerlo.

\section{Funciones de las unidades de información en el contexto actual}

Las unidades de información, llámense bibliotecas, archivos, centros de documentación o información, desarroIlan un funcionamiento cíclico que se inicia con la identificación de las necesidades de conocimiento de sus usuarios reales y potenciales, para luego pasar a la búsqueda/localización de fuentes de información y conocimiento cónsonos con las necesidades detectadas en cuanto a formato, tipo, idioma, presentación, profundidad, entre otros. Posteriormente, es preciso desplegar el procesamiento de la información ubicada y esto constituye el valor agregado, que se materializa en la elaboración de productos informacionales derivados de la aplicación de proce- sos de análisis, los cuales incluyen la identificación de descriptores o palabras clave, la elaboración de resúmenes de distintos tipos, construcción de cuadros comparativos, estadísticas, analogías, y otros. En esencia, con estos procesos se procura reconfigurar los elementos hallados, hacerlos más accesibles desde el punto de vista cognitivo, para generar nuevas lecturas y comprensiones y hacer más viable la creación de conocimiento por parte del usuario.

Con la finalidad de obtener una retroalimentación de su funcionamiento, las unidades de información también han de instaurar mecanismo de evaluación permanente acerca del alcance y efectividad de sus acciones, el grado de satisfacción de su comunidad de usuarios, el surgimiento de nuevas demandas o exigencias intelectivas que hagan necesario el replanteamiento o diversificación de los servicios de información que se ofrecen.

Al considerar la trayectoria histórica de las unidades de información, parece existir mayor énfasis en la conservación física de los materiales en los cuales se encuentra registrada la memoria de la humanidad, a la vez que propician un adecuado acceso a la misma, y esto a pesar del auge que ha tomado el contenido (la información) sobre el continente (el soporte documental). Sin embargo, la importancia otorgada a los soportes de información, considerados como los registros del pasado que garantizarán a los ciudadanos de generaciones futuras el conocimiento de las vivencias y logros de sus antecesores, forma parte de los objetivos funcionales de las unidades de información. 
En este devenir de transformaciones, las funciones de las unidades de información también han sufrido cambios, sus espacios destinados para el almacenamiento de soportes documentales, pasan ahora a convertirse en espacios mediadores del conocimiento, según lo señala Pirela (2004), su acción se orienta a potenciar el aprendizaje tecnológico-informativo de sus usuarios, enfatizando para ello en lo comunicativo y cognitivo. Es decir, que se erigen como formadoras de individuos en el uso de medios tecnológicos para seleccionar, evaluar, sintetizar y compartir contenidos, de manera que puedan emplear estratégicamente el conocimiento disponible, y por ende, logren impactar positivamente el entorno social en el que se encuentran insertos.

\section{Las redes en el contexto de las organizaciones}

La creación de las redes está asociada al valor que ha asumido el conocimiento como recurso inmaterial de fuerte incidencia en la productividad de las organizaciones. En tal sentido, las redes vienen a constituir un mecanismo idóneo para compartirlo y optimizarlo, Vega (2004:4) las define como "estructuras organizativas complejas, formadas por organizaciones relacionadas, que comparten propósitos, funciones y procesos...; y dirigen sus esfuerzos hacia el logro de objetivos comunes".

Las redes nacen para formalizar el establecimiento de relaciones sinérgicas entre las organizaciones, lo que respon- de a la necesidad de compartir recursos, complementarse mutuamente, obtener ventajas competitivas, lograr el acceso a bienes y servicios, alcanzando así mayores márgenes de desarrollo y el incremento del capital social, el cual surge de las relaciones que establecen los actores que integran la institución con otros actores, de manera que este capital se concreta en los productos que se generan a partir del intercambio dado. En la medida que dichas relaciones son más diversas, el capital social se hace más satisfactorio por cuanto permite acceder a las ventajas competitivas necesarias para alcanzar el éxito organizacional.

Burt (2000) señala que las redes se hacen necesarias para desarrollar la capacidad de aprendizaje de las organizaciones, por consiguiente, su configuración debe estar apoyada por las políticas de funcionamiento, con el propósito de que se establezca un clima de confianza que es crucial cuando se plantea un intercambio redificado. El autor indica además que dicha capacidad inicia su evolución valorizando lo nuevo, la información externa y buscando los mecanismos para absorberlos y aplicarlos.

Toda red debe tender a un crecimiento y/o expansión progresiva, en el sentido de ampliar los límites de su alcance, y con ello la inclusión de organismos o personas clave, cuya contribución enriquezca el capital social de todos los miembros, y permita obtener mayores éxitos en las gestiones de cada uno. Aunado a esto, las redes permiten también la identificación de pares (individuos que realizan trabajos simila- 
res en diferentes contextos), y ello hace posible crear marcos de referencia y guías de acción, conducentes a legitimar prácticas, enriquecer esquemas de funcionamiento y otros.

En esencia, los beneficios que se derivan de las redes son según Burt (2000) la información y el control. La primera constituye el conocimiento de las oportunidades existentes en el mercado, su ubicación y la identificación de los participantes; $y$ se determina en función del acceso, el tiempo oportuno y el referente. En cuanto al control, viene dado por el establecimiento de normas, acuerdos o lineamientos entre los miembros de una red, los cuales buscan brindar beneficios a todos; y por medio de la interacción es posible monitorear las acciones de otros y el cumplimiento o no de tales disposiciones.

Las organizaciones pueden encontrar en las redes un espacio para propiciar el crecimiento continuo, para expandir las posibilidades, aprovechar las oportunidades, aprender y desaprender prácticas; todo ello en función del intercambio con otros entes de los cuales puede obtener y ofrecer estímulos, ideas, respuestas, contactos, experiencias; en resumen, las redes permiten que el funcionamiento organizacional se realice articuladamente con el contexto social.

\subsection{Concepción reticular en el funcionamiento organizacional}

Sequeira (2001:15) afirma que en el momento presente "las organizaciones se conciben como campos donde se razonan las acciones, se piensa y se comparten los significados, y además se propician formas más dinámicas de actuar y pensar". Por consiguiente, las funciones asignadas a cada miembro de una organización constituyen canales a través de los cuales puede fluir su conocimiento y creatividad, que unida a la sabiduría de otros, hace posible construir la plataforma innovadora de la organización.

En este sentido, las redes constituyen un espacio de encuentro de sujetos implicados en el alcance de objetivos comunes, este intercambio les lleva a obtener aprendizajes, a compartir experiencias, a desarrollar competencias, ofrecer y recibir colaboraciones, intercambiar roles, entre otros; todo ello mediante la socialización con las TIC.

Suárez (2001) señala que en virtud de la globalización, las redes son consideradas como estrategias para la efectiva negociación en la que día a día se ven envueltas las organizaciones, pues en ellas convergen una pluralidad de actores individuales y colectivos cuyos aportes viabilizan la adecuada adaptación a los cambiantes requerimientos del mercado y del entorno. En estos momentos, las redes se erigen como la organización de organizaciones, es decir, como la estructura que responde a la necesidad de disponer de una plataforma de conocimiento flexible, virtual, heterogénea, capaz de integrar aportes creativos derivados de diversos segmentos institucionales, cuyo procesamiento se realiza eficazmente debido al uso de las TIC, lo que permite a las organizaciones asumir con rapidez los cambios, aminorar los niveles de incertidumbre y por ende mantener vigente su competitividad. 
La dimensión organizacional de las redes se fundamenta en el hecho de que a través de ellas, es posible desplegar un entramado de vínculos estratégicos que conllevan al acceso de una mayor cantidad de conocimiento y a fundamentar relaciones de cooperación y competencia articuladas mediante la comunicación y flexibilidad que caracteriza su funcionamiento.

A medida que aumenta la incertidumbre en los entornos sociales, se originan iniciativas que coadyuvan a mantener el control y a desarrollar un margen de prospectiva que permita incorporar las competencias e innovaciones necesarias; en el presente histórico, las redes cumplen ese cometido, por cuanto imprimen a las organizaciones el dinamismo, la capacidad de adaptación y respuesta a los continuos cambios que se traduce no sólo en su mantenimiento, sino también en el progresivo mejoramiento de su posición competitiva. Debido a la importancia que ha venido cobrando el conocimiento como recurso base para accionar con eficiencia y efectividad, las organizaciones han ido desplazando sus esquemas de funcionamiento hacia formas redificadas, basadas ya no en la mera acumulación de conocimiento (Márquez, 2001) sino en la compartición del mismo.

Por esta razón, las organizaciones en la presente coyuntura posmoderna, se han configurado como conglomerados de gente innovadora cuyas capacidades se canalizan a favor de la adecuada interpretación de las oportunidades, amenazas, fortalezas y debilidades, con miras a generar la mayor competitividad posible. Esto plan- tea nuevos esquemas de funcionamiento organizacional, basados primordialmente en la interacción, pero no con una óptica simplista de seguimiento de patrones rígidos, sino con una visión compleja que admite fracturas de lineamientos, el surgimiento de nuevas rutas, es decir, en los que impera la dialéctica, señalada por Morgan (1998), como el encuentro de los opuestos.

Desde esta perspectiva, el funcionamiento reticular es parte del fundamento esencial en que se apoyan las organizaciones involucradas en una visión de conocimiento, ya que a partir de éste es posible obtener la complementariedad y armonía contextual necesarias para proyectarse en el entorno. Cuando se asume la reticularidad en la organización se abren las puertas al intercambio multidireccional de conocimiento, y al mismo tiempo se promueve un aprendizaje deliberado, expresado en adopción de procesos, formas, implementación de adaptaciones particulares y otros, lo que al final de cuentas constituye innovación, y por ende creación de conocimiento, según lo plantea Rogers (1995).

La redificación del funcionamiento organizacional imbuye al sujeto en una atmósfera de libertad creativa, de comunicación sin barreras, que le lleva a proyectar su acción más allá de las fronteras espacio-temporales de la empresa en la cual labora. Ello debe despertar en él un sentido de pertenencia y compromiso más profundo, al saberse miembro de un colectivo de mayor alcance, en el que sus aportes y el de sus pares no expresan la suma de individualidades sino el producto de una sinergia sustancial con- 
formada por la activación de los procesos creativos de un conjunto de seres pensantes que buscan desarrollar en el presente mayores ventajas competitivas, y en el futuro ser congruentes con los escenarios posibles que plantee el devenir histórico (Rojas, Torres y Arapé, 2001).

La reticularidad constituye el eje del funcionamiento organizacional, el cual se caracteriza por el dinamismo, horizontalidad, flexibilidad, desterritorialidad, confianza, adaptabilidad, multi y transdisciplinariedad y cooperación. A través de esta, las organizaciones se asumen como individuos corporativos inmersos en un continuo aprendizaje e innovación, y por ende, permeables a la configuración y reconfiguración de sus prácticas.

\subsection{Creación y transferencia de conocimiento a través de las redes}

El propósito fundamental de la creación y transferencia de conocimiento es la generación de invenciones e iniciativas que mejoren el posicionamiento de la organización en el entorno global en el cual compite, por ende, la dimensión subjetiva del individuo, que de otra se marginaba para impedir que de alguna manera interfiriera en el seguimiento lineal de pautas o directrices, ahora se libera y se asume como ente impulsor de cambios. En tal sentido, las redes constituyen la plataforma a partir de la cual los sujetos pueden acceder y compartir el conocimiento que requiere su rol productivo.

En virtud de esto, la creación de conocimiento involucra adentrarse en la esfera subjetiva, en el ámbito de las ideas, los ideales y valores de los individuos, y también en lo social, tal como lo señalan Nonaka y Takeuchi (1999). Cuando se considera la complejidad que atañe navegar en el bagaje experiencial, afectivo, cognitivo y emotivo de los seres humanos, resulta muy necesario contar con formas y estrategias novedosas alejadas de toda rigurosidad, aún más cuando se pretende de manera deliberada materializar ese conocimiento en algún tipo de beneficio que rinda ganancias a cualquier organización. Es por ello, que al hablar del contexto o ambiente en el que se ha de propiciar la creación del conocimiento se han de considerar diversos aspectos, por supuesto que todos apuntan al individuo, por cuanto su conocimiento tácito constituye el factor primordial para construir innovaciones.

A partir de lo señalado, la captura de este tipo de conocimiento cobra fundamental importancia, ya que representa el anclaje oculto del cual se desprende el pensar y, por ende, el accionar del sujeto, que además encubre una serie de habilidades, experticias y concepciones fundamentales. En la creación de conocimiento el manejo de la dimensión personal se hace inevitable, es preciso explorar el know how particular, trabajar el sentido de pertenencia organizacional y la visión particular; de modo que la comunicación asume un papel protagónico, en tanto que se intensifica la interrelación entre los miembros de las organizaciones, con el fin de hacer explícito lo implícito y diseminar ampliamente el conocimiento aportado por cada persona. En este punto, es donde las redes intervienen como estructuras viabilizadoras del intercambio que es nece- 
sario suscitar entre los individuos para potenciar las iniciativas creativas que pueden aflorar en ellos.

A través de las redes es posible construir una matriz en la cual converjan los conocimientos particulares de sujetos involucrados en áreas creativas semejantes o complementarias, a partir de la cual se puede tejer un entramado cognitivo más complejo, que utilizado ventajosamente propicia la constitución de una plataforma para desarrollar ventajas competitivas derivadas de las innovaciones.

Von Krogh, Kazuo, y Nonaka, (2001) indican que la creación de conocimiento en las organizaciones requiere identificar el bagaje de experiencias y habilidades particulares de cada sujeto; así como disponer de la información de carácter público que pueda ser de utilidad, también es preciso activar el funcionamiento de las redes, las cuales movilizan multidireccionalmente los activos de conocimiento a las diversas áreas de la organización y hacen posible su aplicación para agregar valor y capitalizar las oportunidades de negocios. A través del intercambio de datos, informaciones y conocimiento, los sujetos inmersos en la creación de innovaciones y aún los que pretenden mejorar sus prácticas, podrían construir esa cohesión cognitiva necesaria para crear y justificar conceptos novedosos, elaborar prototipos e internivelar el conocimiento nuevo y existente.

\section{El valor agregado de las unidades de información en los procesos de transferencia y creación de conocimiento organizacional}

Para la creación de conocimiento es preciso disponer de varios insumos, entre ellos: una plataforma de información confiable y actualizada, la motivación y capacitación suficiente de los individuos involucrados, la voluntad política de la organización que provee los recursos, los medios tecnológicos para viabilizar el procesamiento y el intercambio necesario. El aporte que las unidades de información pueden hacer en dicho proceso posee dos dimensiones: una de tipo informativo-cognitiva y la otra formativa, las cuales apuntan hacia los dos primeros requisitos señalados.

En cuanto a la primera, se tiene que la misión social de las unidades de información se orienta a ofrecer servicios dirigidos a satisfacer necesidades informativas y de conocimiento en los individuos, por lo general, los contenidos que proporcionan se refieren a temáticas específicas o poseen un origen común, es decir, se generan por las acciones de entes particulares, privados 0 públicos. Todos ellos pueden ser utilizados para múltiples finalidades en diferentes contextos.

Los centros de documentación y las bibliotecas especializadas ofrecen información en áreas del conocimiento específicas, propiciando con ello la actualización cognitiva de sus usuarios 
quienes por lo general son estudiosos, investigadores o especialistas en determinados ámbitos de la ciencia; de manera que al establecer contacto con dichos contenidos, y además cuando éstos son dinamizados a través de recursos telemáticos que borran las barreras geográfico-temporales, se establece un cuerpo colegiado de expertos que no sólo intercambian sus saberes entre si, sino que también fortalecen sus conocimientos accediendo a contenidos actualizados y seleccionados en función de intereses intelectuales específicos. Esto lo corrobora la UNESCO (2005), al señalar que las bibliotecas constituyen un pilar de la circulación social de los conocimientos y un factor de vitalidad para las redes; por cuanto son aglutinadores de contenidos que poseen relevancia, actualidad y pertinencia ya que su selección, de entre el universo cada vez más abundante de fuentes, obedece al seguimiento de políticas de evaluación y adquisición, concebidas por profesionales en el área de la información cuya formación les permite aplicar estrategias adecuadas para hacer elecciones más acordes con las necesidades de los usuarios.

Por otra parte, los contenidos que proveen los archivos de empresas, instituciones públicas o de particulares, también poseen una riqueza testimonial importante, que corresponde al conocimiento explícito que se genera al interior de las mismas, es decir, aquel que ha sido formalmente estructurado y registrado (Nonaka y Takeuchi, 1999), que además deja constancia de las decisiones tomadas, del funcionamiento y rendimiento organizacional o particular. De esta conjugación de datos e informaciones se pueden elaborar síntesis, resúmenes, estadísticas, proyecciones, revisiones retrospectivas, y otros, que constituyen productos informacionales derivados de las funciones que cumplen las unidades de información archivísticas.

El contenido de dichos productos tiene la particularidad de poseer mayor objetividad y carácter orgánico, es decir, tiende a alejarse de opiniones y valoraciones, mientras que se acerca a reflejar la cotidianidad funcional de un ente individual o colectivo. A la vez, posee continuidad con los antecedentes y consecuentes, porque en conjunto reflejan una sucesión cronológico-orgánica de hechos. De manera que sobre la base de éstos contenidos, se pueden tejer infinidad de análisis que coadyuven a conocer alguna realidad determinada o bien a identificar factores influyentes, relaciones causa-efectos, desviaciones, entre otros.

Cuando los productos informacionales derivados del análisis documental aplicado en las unidades de información archivística, son movilizados redificadamente pueden llegar a constituirse en importantes aportes intelectuales, en tanto que mediante ellos es posible dibujar escenarios posibles para la toma de decisiones basados en experiencias reales, evaluar resultados/consecuencias de algún curso de acción, medir el alcance y la efectividad de procedimientos, métodos o estrategias administrativo-gerenciales en particular, obtener visiones retrospectivas y actuales de entes reales que pueden servir para emular o evitar comportamientos. En líneas generales, los contenidos que las unidades de archivos pueden poner a disposición en las redes de conocimiento 
tiende al enriquecimiento del aprendizaje, además que incorpora insumos que pudieran contribuir a generar nuevas propuestas, y por ende, a impulsar la creatividad de los participantes de las mismas.

La contribución informativo-cognitiva de las unidades de información en los procesos de transferencia y creación de conocimiento, está dado por el suministro de contenidos cuya validez, confiabilidad, actualidad y pertinencia ha sido anteriormente evaluada por profesionales en el área de la información, lo que constituye un valor agregado en tanto que en la Web circulan muchos datos no estructurados y redundantes, información inconsistente y repetida, es decir, de baja calidad, porque no siempre se someten a un proceso editorial mínimo que regule su relevancia, veracidad, profundidad y otros rasgos (García, 2002).

Se podría decir que en éste sentido, las unidades de información ejercen una función de arbitraje de contenidos, lo cual fortalece la plataforma informativocognitiva sobre la cual trabajan las redes de conocimientos, cuyos miembros requieren acceder a datos e informaciones pertinentes, desde el punto de vista de la temática, valor, fiabilidad y actualidad. En la medida que se verifica la confiabilidad integral de los contenidos que manejarán los participantes en la construcción del conocimiento, en esa misma proporción crecen las posibilidades de generar importantes contribuciones intelectuales en la búsqueda de soluciones a problemas o la creación de invenciones. Visto de desde una perspectiva sistémica, si se elevan los niveles de calidad en los insumos de entrada aumentan las probabilidades de obtener un mejor producto de salida.

Además de la evaluación/validación de los contenidos, es necesario mencionar también la estructuración y sistematización de los mismos, que constituye otro de los aportes dentro de la dimensión informativo-cognitiva que pueden realizar las unidades de información. Porque aunque se manejen elementos inmateriales, es preciso establecer alguna metódica de presentación que responda a las posibles lógicas de búsquedas empleadas por los usuarios; a la vez que les permita conocer las relaciones temáticas, jerárquicas 0 asociativas que pueden existir entre ellos, esto con el fin de evitar combinaciones disonantes de datos o informaciones, originadas por el desconocimiento de su procedencia o naturaleza teórica.

En esencia, las unidades de información pueden facilitar la aprehensión intelectual de los contenidos de interés a los participantes de las redes de conocimiento, propiciando un acceso cómodo así como el manejo más inteligente y metódico de los contenidos, lo cual apoya el proceso de innovación que deben adelantar las organizaciones competitivas.

Con respecto a la dimensión formativa del aporte de las unidades de información al proceso de creación de conocimiento, puede decirse que se relaciona con la alfabetización informacional, mediante la cual los individuos resultan capacitados para entresacar del universo informativo inagotable los constructos cognitivos que requieren para hacer más productiva su labor. La 
importancia de dicha alfabetización crece en la medida que el volumen de los contenidos tanto digitales como impresos, se incrementa vertiginosamente haciéndolos cada vez más inaccesibles desde el punto de vista intelectual por cuanto se hace imposible captarlos, filtrarlos y mucho menos aprehenderlos cognitivamente.

La alfabetización informacional gira en torno al aprendizaje de aquellos procesos que permiten intervenir la información propiamente dicha, para crear conocimiento; así como al desarrollo de actitudes críticas necesarias para conocer la ideología que sustenta las diferentes propuestas, desentrañar los valores, finalidad que persiguen, sus principales destinatarios (Díez, 2001). $Y$ se sustenta en el hecho de que la información en si misma resulta de poco valor si las personas no tienen las habilidades para encontrarla y luego valorarla críticamente (Byrne, 2005); de manera que, aunque la información es un factor de producción, es preciso desarrollar competencias específicas referidas al ámbito informacional, que capaciten para gestionar efectivamente diversos tipos de contenidos con la finalidad de construir conocimientos.

La alfabetización en información se hace altamente necesaria cuando el hallazgo, uso y la validación de información constituye un reto permanente para innovar, corregir irregularidades, desarrollar procesos de negocios, gerenciales, técnicos, y otros (Ramírez, 2001; Vieria, 2004). Es definida por Cuevas y Vives (2005:58) como "el desarrollo de habilidades para buscar, recuperar, evaluar y usar la información mediante instrumentos intelectivos del método científico y técnicos informáticos"; por su parte, Byrne (2005) señala que es el conjunto de capacidades, conductas y actitudes necesarias para acceder, valorar y utilizar la información de manera eficaz en la vida diaria.

De estas definiciones se entiende que la alfabetización en información persigue como objetivo fundamental capacitar al individuo como un gestor efectivo de los diversos recursos de información que tiene a su disposición en el momento presente; como un analista-sintetizador, experto en la deconstrucción y construcción de los discursos existentes en las distintas modalidades y formatos; y como un creador-aplicador de conocimientos, que garantice su desempeño social de alto impacto positivo en el entorno que le rodea. En esencia, la alfabetización en información pretende otorgar a los usuarios de la información, mayor capacidad de desenvolvimiento independiente en las búsquedas de la información que consideren necesarias para mitigar sus necesidades informativas, y propiciar a la vez su crecimiento cognitivo.

La alfabetización informacional incluye la ejercitación y profundización del individuo en los procesos de interpretación, evaluación y reflexión, mediante los cuales le es posible transformar la información en nuevos conocimientos, desarrollar sus capacidades cognitivas, es decir, el aprender a aprender, el aprender independiente/autónomo, promover el ingenio y la creatividad derivados de la información 
que maneja, internalizar los valores de la información; Cuevas y Vives (2005) le añaden a este tipo de alfabetización la destreza de saber comunicar la información o el conocimiento elaborado, ya que esto le permite al hombre participar activamente en la sociedad.

Las unidades de información contribuyen a desplegar la alfabetización informacional desde diversos puntos de acción: viabilizando la construcción de hábitos lectores y de aprendizaje; ayudando a sus usuarios por un lado, a manejar estratégicamente la heterogeneidad producto de los múltiples tipos de medios, formatos, lenguajes y alfabetos (García, 2002); y por el otro, a descubrir lo que realmente necesitan saber, es decir, capacitándolos para identificar sus necesidades y expresarlas de una forma que les sea fácil encontrar lo que requieren, esto demanda una interacción efectiva con los sistemas de recuperación de información que incluye la especificación de las preguntas, la interpretación de las respuestas obtenidas, el manejo de las respuestas amplias, la clasificación y selección de los documentos que resultan válidos, el rastreo efectivo de contenidos; por último, dicha alfabetización tiende a propiciar la comprensión, asimilación y creación del conocimiento necesario (Byrne, 2005; Cuevas y Vives, 2005; Gómez y Licea, 2005).

La alfabetización informacional que han de desarrollar las unidades de información puede verse como un servicio evolucionado, que incluye la enseñanza de las competencias para acceder y usar la información con el fin de crear conocimientos y transmitirlos, utilizando medios convencionales o electrónicos; y también debe considerar la instrucción bibliográfica que le va a permitir a los usuarios apreciar y orientarse en la multitud de fuentes que contribuyen a enriquecer su conocimiento, específicamente el acervo del que disponen tanto las bibliotecas tradicionales como las electrónicas, el cual resulta bastante beneficioso para complementar su aprendizaje (Bawden, 2002; Gómez y Licea, 2005).

El aporte formativo que las unidades de información pueden realizar en las redes de conocimiento se concreta como una especie de servicio de asesoramiento a sus participantes, para propiciar un mejor acceso a los contenidos de interés, y al procesamiento intelectual de los mismos. En tal sentido, las unidades de información previa elaboración de un estudio o sondeo acerca de las principales necesidades presentes en los miembros de las redes, pueden desarrollar planes de capacitación en áreas específicas del ámbito informacional, bien sea en la identificación de sitios web o portales de interés en temas determinados, así como la valoración de fuentes de información, elaboración de resúmenes o síntesis, identificación de elementos esenciales de la estructura discursiva, ejecución de análisis de información y sus diversos tipos, entre otros; los cuales resultan esenciales para simplificar el tiempo y esfuerzo intelectual (lectura, comprensión, interpretación, aplicación) que invierten los individuos para crear conocimiento.

En líneas generales, los cambios que ha traído consigo el "boom" tecnológico han hecho que las unidades de 
información pasen de contenedoras y facilitadoras del conocimiento explícito, a mediadoras desde una doble perspectiva. En primer lugar, porque continúan de alguna manera siendo el interfaz entre el conocimiento explícito y los usuarios de este; en segundo lugar, porque ejercen también una labor educativa propiciando en los sujetos el desarrollo de habilidades para actuar autónomamente en el hallazgo y análisis de fuentes de interés. Al respecto, Pirela (2004) arguye que las unidades información contribuyen a la consolidación de una noción de conocimiento articulada desde la mediación, cognición y aprendizaje, viabilizada mediante los procesos de organización, representación, estructuración, contextualización y análisis crítico de la información significativa y pertinente para usuarios específicos; en esencia, las unidades de información propician nuevas formas de acercar el saber a los usuarios, para que estos construyan individual y colectivamente soluciones e innovaciones que generen riquezas y bienestar social.

\section{Las unidades de información: entes de apoyo a las redes organizacionales}

Las bibliotecas, archivos, centros de información y documentación asumen la responsabilidad de desarrollar infraestructuras informativas que propicien la actualización de conocimientos mediante el intercambio de ideas y el aprendizaje continuo, asumiendo el rol de centros de conocimiento o diseñadores de comunidades inteligentes, que apoyan el trabajo de las redes organizacionales (Sequeira, 2001).
Para cumplir este cometido las unidades de información asumen una dimensión más virtual que material, en el entendido que no se circunscriben solamente a colecciones documentales físicas, las que además continúan siendo útiles en el potenciamiento intelectual de los miembros de las organizaciones; sino que funcionan bajo una concepción reticular armonizando con el ciberespacio y a la vez con la información particular que genera o posee la organización. Puede indicarse que parte de su rol es el de facilitar y a la vez normalizar las formas de acceso, búsquedas y análisis de la información requerida, velando por su calidad, confiabilidad, y la adecuada infraestructura para el manejo de esta. Su énfasis está en descubrir, mediante un monitoreo permanente; capturar, a través de una acertada selección; enviar proactivamente (si es posible antes de ser requeridos); y presentar adecuada y consistentemente (utilizando metadatos, formatos, lenguajes documentales, etc.), los recursos cognitivos que los miembros de las redes organizacionales requieren para mejorar su actuación presente y futura.

Las unidades de información apoyan el funcionamiento de las redes en cuanto se erigen como entes suministradores de recursos informativos en diversas presentaciones, gestionando su ubicación y acceso, asesorando acerca de las nuevas formas de estructurar y leer los documentos de esta generación que han asumido cambios drásticos en cuanto a su presentación y contenido.

Además de esto las unidades de información también ejercen una labor de enseñanza, a través de la formación de 
usuarios, autónomos y críticos, ya que tal como lo señalan Gazpio y Álvarez (1999), enseñar a aprender implica enseñar a informarse, y ello no solamente se refiere a la ubicación de datos, informaciones o conocimientos de interés, sino también el desmontaje y descontrucción de contenidos nuevos o existentes. Evidentemente que esto se relaciona con lo que señalan Von Krogh, et al (2001) en cuanto al mejor uso del conocimiento existente, y el diseño de mejores formas de aprovechar lo que se sabe.

El aporte de las unidades de información forma parte de la base de la estructura de intercambio y mediación que las redes propician, por cuanto constituyen nichos intelectuales que enriquecen la construcción del conocimiento por parte de los actores involucrados, abriendo espacios para el hallazgo oportuno, y el uso idóneo de las fuentes de información. Vistas de éste modo, a las unidades de información es posible considerarlas como entes asesores en el acceso y procesamiento de recursos de conocimiento, haciendo posible una aproximación constante entre el universo, cada vez más extendido, de dichos recursos, y el sujeto creativo.

Desde una óptica corporativa, las redes se asumen como una nueva tipología de individuo-usuario de las unidades de información, configurado a partir del auge que ha tomado la creación de conocimiento y el intercambio telemático de información; sus necesidades cognitivas, delineadas desde los propósitos y objetivos que se plantean, representan un nuevo espacio de acción social. Las unidades de información apoyan y facilitan la ac- tualización intelectual de sus participantes, no sólo al viabilizar el acceso a lo novedoso sino también al impulsar el desarrollo de competencias analítico-tecnológicas en los sujetos que participan en ellas.

\section{Conclusiones}

En su devenir histórico, las unidades de información han ido adaptando sus prácticas cotidianas a los cambios ocurridos, también han asumido otras praxis que en parte devienen de la incorporación de las TIC, las cuales plantean nuevas formas de acceso a los contenidos, el surgimiento de nuevos medios y formatos de presentación, modifican las demandas y preferencias informativas de los usuarios, entre otros. De tal manera, que el funcionamiento de tales entes informativos tiende a complejizarse, en tanto que se hace también más flexible y abierto a las impredecibles necesidades que pueda plantearle el entorno organizacional en el que se encuentren insertas.

Por su parte, la colaboración y el intercambio de recursos entre las organizaciones, propicia el fortalecimiento de las redes como plataforma de interacción que coadyuva al "ganar ganar", en el sentido que permite compartir información y conocimiento, y con ello desarrollar innovaciones y ventajas competitivas necesarias para mantenerse en el mercado global. Mediante las redes, los individuos involucrados en el logro de fines comunes 0 semejantes, desarrollan relaciones sinérgicas, en las que se cohesionan sus aportes intelectuales, y a la vez se 
intercambian contenidos de interés provenientes del entorno o de su propio funcionamiento y que pueden ser utilizados para afectar positivamente su rendimiento.

En esta integración de esfuerzos organizacionales, las unidades de información intervienen apoyando los procesos de transferencia y creación de conocimiento lo que a su vez repercute en el incremento del capital intelectual de la sociedad; su acción se enfatiza en arbitrar, filtrar y facilitar el acceso a fuentes de información idóneas, agregando valor mediante la aplicación de procesos de análisis, síntesis, clasificación, simbolización, y otros. Todo ello, con el fin de disminuir el tiempo y esfuerzo necesarios para el hallazgo de contenidos que realmente potencien la creatividad de los individuos participantes en las redes.

Las unidades de información también se orientan a fomentar en los individuos el desarrollo de competencias analítico-sintéticas para extraer los elementos medulares del contenido de las fuentes a las que se accede. Propiciando de este modo la formación de usuarios cada vez más autónomos en el uso de la información y el conocimiento, y capaces de idear sus propias formas de manejar los contenidos de su interés, basados en las oportunidades y circunstancias en torno a las cuales funcionen tanto las redes como las organizaciones en las que laboran. En tal sentido, las unidades de información deben desarrollar una función pedagógica hacia sus usuarios, y asumir- la como una tarea permanente, que forma parte de su responsabilidad social, y que es además fundamental para que los individuos puedan vivir en esta sociedad compleja y mutante (Campello, 2003).

Las unidades de información incursionan en espacios redificados como coadyuvantes para el efectivo proceso de intercambio, su contribución en tales ambientes revaloriza la acción de cada participante, en el sentido que le ofrece mecanismos para enriquecer sus aportes de conocimiento a la red, a la vez que impulsa el incremento de su potencial creativo proveyéndole herramientas cognitivas para el acceso y el análisis de las fuentes de información. En resumidas cuentas, el aporte de las unidades de información a las redes organizacionales se orientan a potenciar intelectivamente al sujeto, para que su actuación sea cada vez más valiosa en la creación de conocimiento y en la generación de innovaciones, contribuyendo de este modo a la consolidación de una sociedad basada en el conocimiento y en las TIC con sentido incluyente, mejorando el aprovechamiento y uso de sus recursos por parte de los usuarios, aportando valor agregado a la información que proveen y ayudando a conservar los valores democráticos.

\section{Referencias Bibliográficas}

Almirón, Núria (2002). Los amos de la globalización. Barcelona: Plaza \& Janés.

Bawden, David (2002). Revisión de los conceptos de alfabetización informacional y alfabetización digital. 
Anales de Documentación, (5), 361408.

Burt, Ronald (2000). The network structure of social capital. Chicago: University of Chicago and Institute Européen d'Administration d'Affaires.

Byrne, Alex (2005). La alfabetización informacional desde una perspectiva global: El desastre agudiza nuestras mentes. Anales de Documentación, (8), 7-20.

Campello, Bernadete (2003). O movimiento da competencia informacional: Uma perspectiva para o letramento informacional. Ciencia da Informaçao, 32(3), 1-17.

Cuevas, Aurora y Vives, Josep (2005). La competencia lectora en el estudio PISA. Un análisis desde la alfabetización en información. Anales de Documentación, (8), 51-70.

Díez, Enrique (2001). Dimensiones críticas de la nueva sociedad del conocimiento. Ponencia presentada en el Congreso Internacional de Tecnología Educación y Desarrollo Sostenible, Murcia, España.

García, Antonio (2002). Organización y gestión del conocimiento en la comunicación. Asturias: Trea, S.L.

Gazpio, Dora y Álvarez, Marcela (1999). Soportes en la biblioteca de hoy. Buenos Aires: CICCUS

Gómez José y Licea, Judith (2005). El compromiso de las bibliotecas con el aprendizaje permanente. La alfabetización informacional. En: Pedro López y Javier Gimeno (coords.), Información, conocimiento y bibliotecas en el marco de la globalización neoliberal (pp.145-177). Asturias: TREA, S.L.

Kerckhove, Derrick de (1999). La piel de la cultura. Barcelona: Gedisa.

Márquez, María del Valle (2001). Nuevas tecnologías, nuevas organizaciones, nuevos aprendizajes. En: Rigoberto Lanz (comp.), Organizaciones transcomplejas. (pp.145-160). Caracas: IMPOSMO/CONICIT.

Morgan, Gareth (1998). Imágenes de la organización. Madrid: RA-MA.

Nonaka, Ikujiro y Takeuchi, Hirotaka (1999). La organización creadora de conocimiento. México: Oxford University Press.

Padrón, José (1996). ¿Qué es un problema de investigación?. En Chacín, M. y Padrón, J. Investigación-Docencia, Temas para Seminario. Caracas: Publicaciones del Decanato de Postgrado de la USR.

Pirela, Johann (2004). Los procesos de mediación en organizaciones de conocimiento de la cibersociedad. Tesis doctoral mención publicación, Universidad del Zulia, Venezuela.

Ramírez, Elsa (2001). La lectura: Un problema para la sociedad de la información. Investigación Bibliotecológica 15(31), 95-211.

Rogers, E (1995). Difusión of Innovations. (4a. ed.). New York: The Free Press.

Rojas, Luis, Torres, Reiny Beth y Arapé, Elizabeth (2001). Posmodernidad: Lógicas organizacionales, lógicas tecnológicas. En: Rigoberto Lanz (comp.), Organizaciones transcomplejas. (pp.23-80). Caracas: IMPOSMO/CO$\mathrm{NICIT}$.

Sequeira, Deyanira (2001). Bibliotecas y archivos virtuales. Santa Fe: Nuevo paradigma.

Suárez, Evalú (2001). Nuevos modelos de gestión. Nuevos modelos organizativos. ¿Organizaciones posmodernas? En: Rigoberto Lanz (comp.), Organizaciones transcomplejas (pp.8196). Caracas: IMPOSMO/CONICIT.

UNESCO (2005). Informe mundial de la UNESCO. Hacia las sociedades del conocimiento. París: UNESCO. 
Vega, Marinela (2004). Redes: interacción organizacional inteligente. Maracaibo: EDILUZ.

Vieira, Silvania (2004). Identificando competencias informacionais. Ciencia da Informaçao, 33(2), 1-16.

Villanueva, Eduardo (1997). El ciberespacio, ¿La frontera final?. En: Hildegardo Córdova (ed.), Espacio: teoría y praxis. (pp. 399-408). Lima: PUCP.
Von Krogh, Georg, Kazuo, Ichijo y Nonaka, Ikujiro (2001). Facilitar la creación de conocimiento. México: Oxford University Press.

Wei, Chun (1999). La organización inteligente. El empleo de la información para dar significado, crear conocimiento y tomar decisiones. México D.F.: Oxford University Press. 